\title{
NIRVANA Network Requirements
}

\author{
Bradley J. Wood \\ Engineering Network Development Division \\ Sandia National Laboratories \\ Albuquerque, NM 87185 \\ July 25,1990
}

\begin{abstract}
NIRVANA is an effort to standardize electrical computer-aided design workstations at Sandia National Laboratories in Albuquerque, New Mexico. The early effect of this project will be the introduction of at least 60 new engineering workstations at Sandia National Laboratories, Albuquerque, and at Allied Signal, Kansas City Division. These workstations are expected to begin arriving in September 1990.
\end{abstract}

This paper proposes a design and outlines the requirements for a network to support the NIRVANA project. The author proposes a near-term network design, describes the security profile and caveats of this design, and proposes a long-tern networking strategy for NIRVANA. 


\section{Contents}

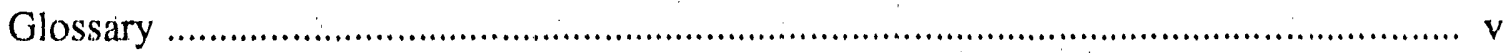

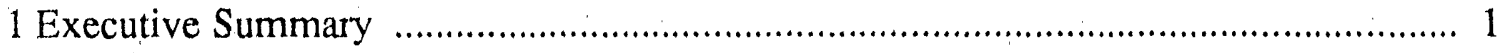

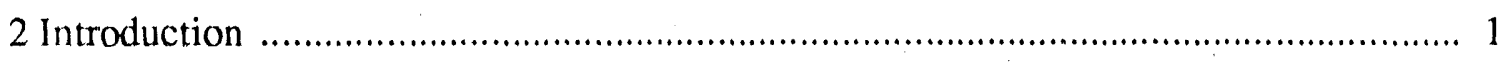

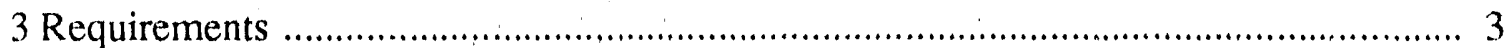

3.1 IEEE 802.3 Physical Connections …......................................................... 3

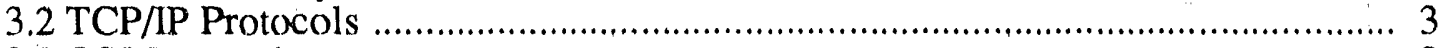

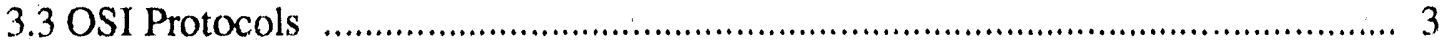

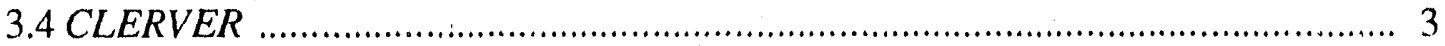

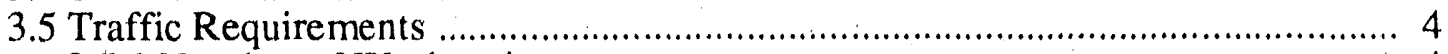

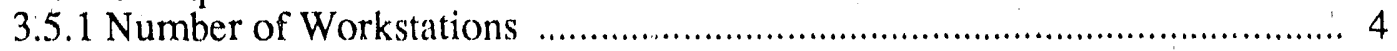

3.5.2 High-Performance Workstations .................................................... 5

3.5.3 Workstation Configurations ….......................................................... 5

3.5.4 File Transfer ................................................................................. 5

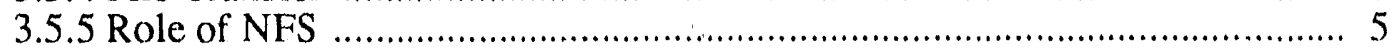

3.6 Access to Other Systems ................................................................................... 5

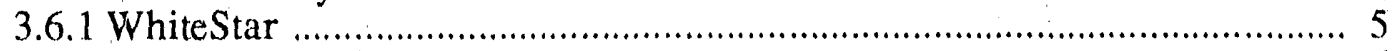

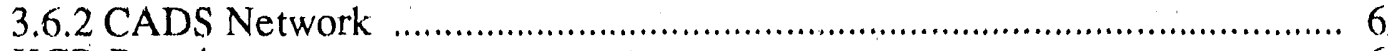

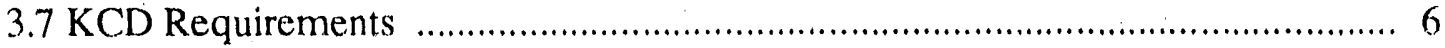

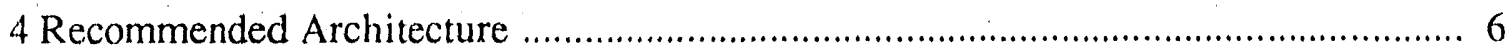

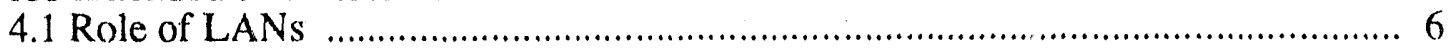

4.2 Fiber-Optic Backbone …............................................................................ 7

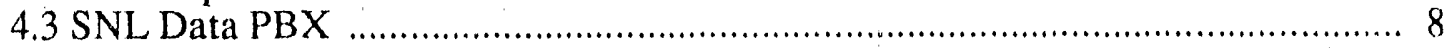

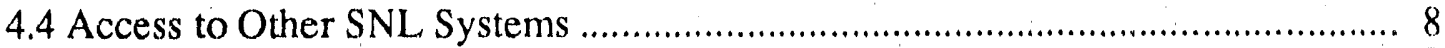

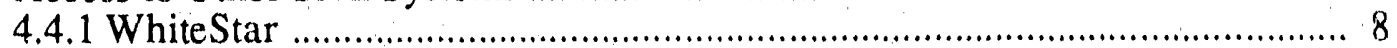

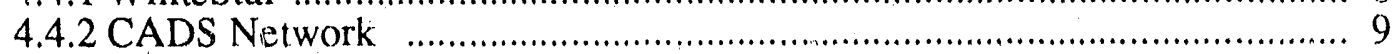

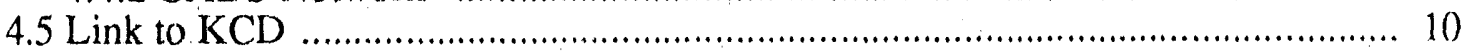

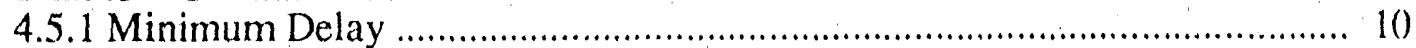

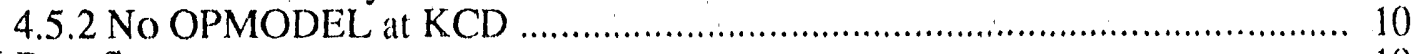

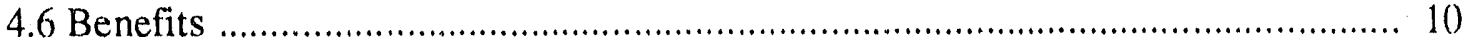

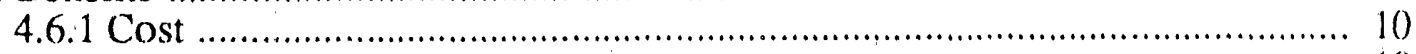

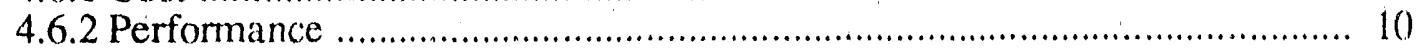

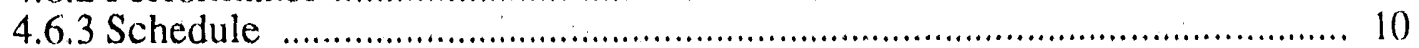

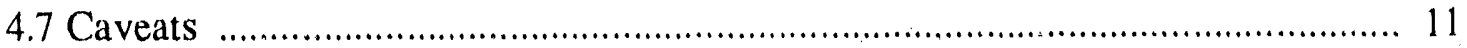

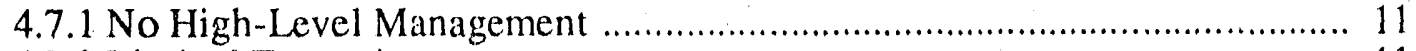

4.7.2 Limited Expansion ........................................................................ 11

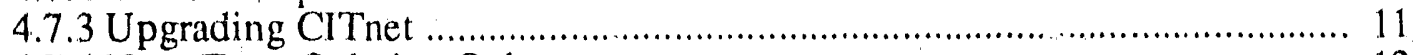

4.7.4 Near-Term Solution Only .............................................................. 12

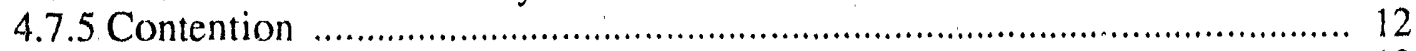

4.7.6 No Classified Processing .................................................................... 12 


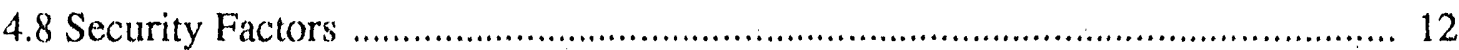

4.8.1 Overall Security Profile ................................................................... 13

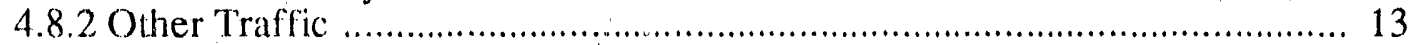

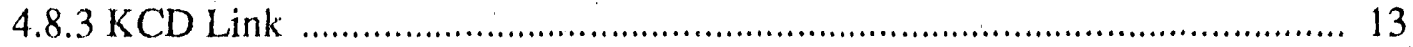

4.8.4 CAD Network Access .................................................................... 13

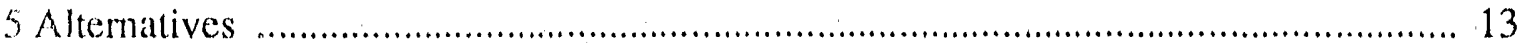

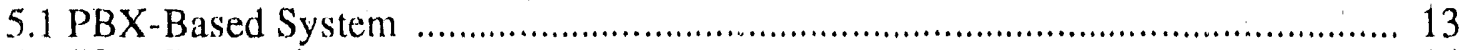

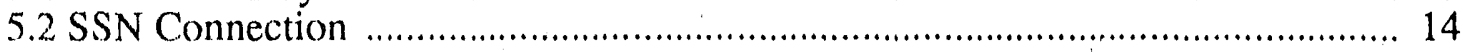

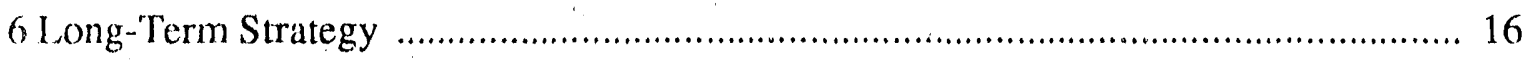

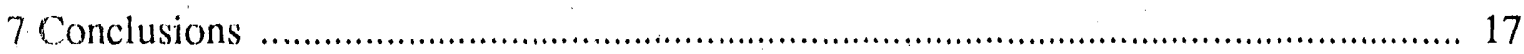

\section{Figures}

Figure 1 - OSI and TCP/IP Network Protocols ............................................................ 2

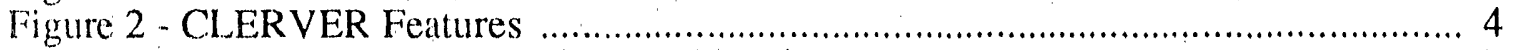

Figure 3 - NIRVANA Network, Geographic View ................................................. 7

Figure 4a - NIRVANA Network, Schematic View ............................................ 8

Figure 4b - NIR VANA Network, Schematic View .................................................... 9

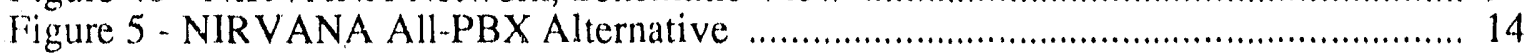

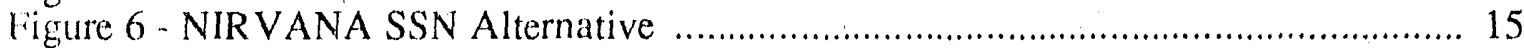

Figure 7 - NIRVANA Long - Term Architecture ..................................................... 16 
NIRVANA Network' Requirements

10Base-2

10Base- 5

10Base-"T

CAD

CADS Network

CCITT

CITnet

CLER.VER

CSMA/CD

DECnet

DECsystem

DNS

DS/3

FDDI

FIPS PUB 146

FIAM

FTP

GOSIP

IEEE 802.3

$\mathrm{K} \cdot \mathrm{b} / \mathrm{s}$

$\mathrm{KCD}$

\section{Glossary}

IELE $8(02.3$ specification for thin coax cable interface, also known as "thin wire"

IEEE $8(02.3$ specification for thick coax cable interface, also known as "thick wire"

IEEE 8()2.3 specification for unshielded twisted-pair cable interface computer-aided design

Computer Aided Design System Network, operated for SNL Organization 280()

Comité Consultatif Internationale de Télégraphique et Téléphonique, an international communications standards body

CAD Integration and Technology Network, operated by SNL Department 2810)

(CLient-sER VER) Xwindows application for sharing workstation windows among multiple users

carrier-sense multiple access with collision detection, a network access method

a proprietary suite of network communications protocols developed by Digital Equipment Corporation

Digital Equipment Corporation's RISC-based family of UNIX workstations and servers

domain name servict:

a digital-signaling statndard for data streams at $45 \mathrm{Mb} / \mathrm{s}$

Fiber-Distributed Data lnterface, a fiber-optic network interface for data streams at $100 \mathrm{Mb} / \mathrm{s}$

Federal Information Processing Standard Publication \# 146, sontco of the GOSIP standard

File Transfer and Management Protocol, an OSI Protocol

File Transfer Protocol, a TCP/IP protocol

Government Open Systems Interconnect Profile, a networking standard for the US Governmen!

Institute of Ekectrical and Electronics Engineers standard for CSMA/CD) networks, also refered to (incorrectly) as Ethernet

thousand bits per second

Allied Signal, Kansas City Division 
LAN

LANmark

LAT

LattisNet

$\mathrm{Mb} / \mathrm{s}$

NIRVANA

NFS

OPMODEL

Organization 2000

Organization 2600

Organization 2617

Organization 2618

Organization 2800

Organization 2810

Organization 2813

Organization 2850

Organization 8000

Organization 9000

OSI

PDS

PBX

RFQ

RISC

SE/VMS

SMTP

SNL local area network

trade name for $960 \mathrm{~Kb} / \mathrm{s}$ IEEE 802.3 network service offered on Intecom Data PBX

local area transport, a virtual terminal network protocol developed by Digital Equipment Corporation

proprietary interface for delivering IEEE 8()2.3 network services over unshielded twisted-pair wiring by SynOptics Communications, Incorporated

million bits per second

project to standardize CAD systems for electrical design

Network File System, a TCP/IP application

satellite based telecommunications network operated for the US

Department of Energy

SNL. Component Development Vice Presidency

SNL Computing Directorale

SNL Computer Communication Design Division

SNL Communications Engineering and Services Division

SNL Design Engineering Directorate

SNL Design and Product Sipport Department

SNL Engineering Network Development Division

SNL, Design Department

SNL Livermore Programs Vice Presidency

SNL Exploratory Systems Vice Presidency

Open Systems Interconned

Protected Distribution System, the internal secure data communications plant at SNL

Private Branch eXchange, a telecommunications switching system

request for quote

reduced instruction set computing

security-enhanced Virtual Memory System, a secure version of Digital Equipment Corporation's VMS Operating System

Simple Mail Transfer Protocol

Sandia National Laboratories 
SNMP

SSN

T1

TCP/IP

WIN/TCP

WhiteStar

X.400/1984

$\mathrm{XNS}$

Xwindows

$\mathrm{VAX}$

VMS
Simple Network Management Protocol

SNL Secure Supercomputing Network

an analog transmission standard for modems that support data streams $1.544 \mathrm{Mb} / \mathrm{s}$

Transmission Control Protocol, Internet Protocol

TCP/IP communications software for VAX systens, a product of The Woliongong Group

SNL electrical component parts database system

1984 CCITT messaging recommendation, an electronic mail standard

Xerox Network System, a suite of network protocols developed by Xerox.

standard for developing windows-based user interfaces

family of minicomputers produced by Digital Equipment Corporation.

Virtual Memory System, a minicomputer operating system produced by Digital Equipment Corporation 


\section{NIRVANA Network Requirements}

\section{Executive Summary}

Sandia National Laboratories (SNL) must construct a network to support NIRVANA workstations. The network must support 41 workstations at SNL, Albuquerque, and 19 workstations at Allied Signal, Kansas City Division (KCD) by April 1991. This same network must be capable of supporting as many as 5()) NIRVANA workstations over the next three years.

We have proposed a near-term design for a NIRVANA network that will expand the existing 2810 development network to satisfy most NIRVANA requirements. This expansion will include a high-performance link between the SNL. NIRVANA network and a KCD NIRVANA local area network (LAN). We can construct this network quickly with minimal costs to provide a secure-unclassified system.

In addition, we have examined two alternatives to building the NIRVANA network. First, we looked at building the network using the SNL data private branch exchange (PBX) as the backbone. This solution is inexpensive, but it does not deliver the needed performance. We also examined a design that uses the SNL Secure Supercomputing Network (SSN) as the NIRVANA backbone. The SSN has many disadvantages for us, but it is the only solution that networks both classified and unclassified workstations.

We have also proposed a long-term network architecture for NIRVANA. This future network would use the emerging Fiber-Distributed Data Interface (FDDI) technologies to deliver excellent performance and security. We could also preserve some of our network hardware in this future architecture. We could build this network over the nexi three years in a joint venture between SNL's Computing and Design Engineering Directorates.

\section{Introduction}

NIRVANA is an effort to set workstation standards for electrical designers and engineers. This project mainly involves SNL Organizations within the Component Development Vice Presidency, Organization 2000), who work with electrical designs. In addition, KCD is also a major player in this effort because they build most electrical designs developed at SNL. Also, SNL. Organizations 8000 and 9000 could soon become NIRVANA partners.

The early effect of NIRVANA will be the introduction of 6() new engineering workstations. These workstations will use common hardware and software platforms. The present schedule calls for delivery of these workstations to begin as early as September 1990. However, this could be just the beginning. Some estimate that NIRVANA could introduce as many as 5()() new workstations at SNL and KCD.

Although much work has gone into defining the workstations' characteristics, little work has gone toward developing a network to support these workstations. Therefore, the NIRVANA consortium has asked SNL's Engineering Network Development Division, Organization 2813, 10 propose a network design.

This plan proposes a basic network architecture. In particular, we will limit our scope to design. ing a network to support Layers 1 and 2 of the Open Systems Intercomnect (OSI) network model 


\section{NIRVANA Network Requirements}

(Figure )1. We will not deal with any higher level protocols except to recognize some specific requirements. The reader should know that a successful network must manage all seven layers of the ISO model. We may address the remaining network layers in a future document.

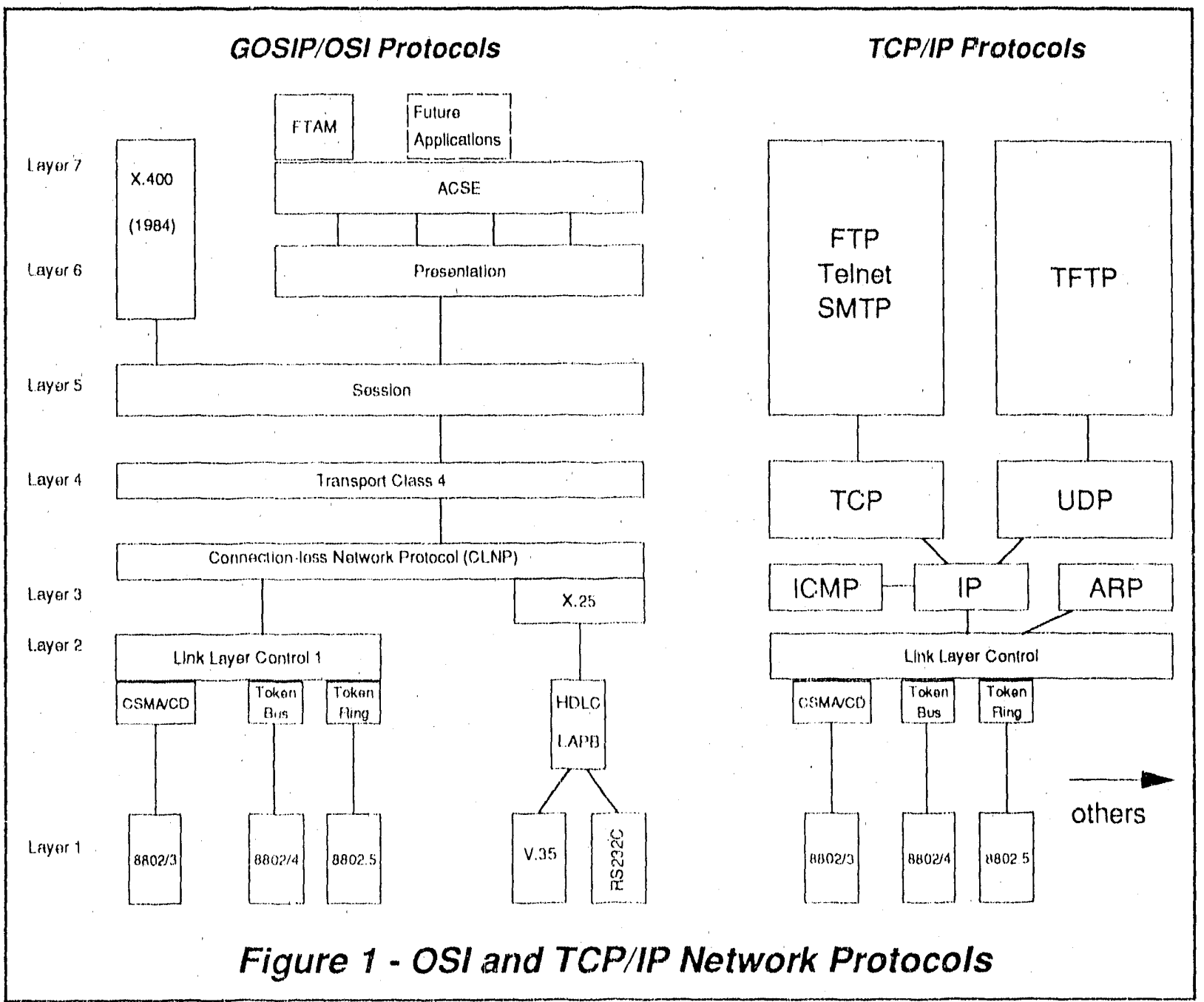

This proposal is not a security plan nor a project plan. The document simply recommends a net. work strategy for consideration by the NIRVANA consortium to stimulate discussion among interested parties. Ideally, the proposal could evolve into a detailed requirements document for a future networking project. 
NIRVANA Network Requirements'

\section{Requirements}

The proposed NIRVANA workstation design drives most network requirements. Although the NIRVANA team has not yet selected a particular workstation or vendor, the team has listed the requirements for these workstations and we will extract them from the NIRVANA request for quote (RFQ).

\subsection{IEEE 802.3 Physical Connections}

All workstations will have an IEEE 802.3 network interface. However, the RFQ does not specify the exact connection for this interface. Therefore, the network should support all common IEEE 8(2.3 connections including 10Base-2, 10Base-5, and 10Base-T.

\subsection{TCP/IP Protocols}

All workstations will include Transmission Control Protocol/Internet Protocol (TCP/IP) network software and must support most common TCP/IP applications and protocols. These applications and protocols include:

- File Transfer Protocol, FTP

- Telnet virtual terminal protocol

- Network File System, NFS

- Simple Mail Transfer Protocol, SMTP

- Domain Name Service, DNS

- Xwindows Release 11.3

- White Pages

- Yellow Pages

\subsection{OSI Protocols}

The NIRVANA network will also follow the Government Open System Interconnect Profile (GOSIP). This requires that all workstations have an ISO-approved network interface. The IEEE 802.3 interface specified in Section 3.1 satisfies this particular recinirement.

The current version of GOSIP also requires that the network support the File Transfer Management (FTAM) protocol and the Comité Cinsultatif Internationale de Télégraphique et Téléphonique (CCITT) 1984 X.400) messinging protocol recommendation. These standards are defined by the Federal Information Processing Standard Publication \# 146 111.

\subsection{CLERVER}

NIRVANA may also embrace a new network technology that SNL Division 2813 is develop.. ing (Figure 2). CLERVER (CLient-sERVER) allows users on multiple workstations to view and manipulate common windows. CLERVER will operate on any display that uses $\mathrm{X}$ windows protocols $|2|$. 


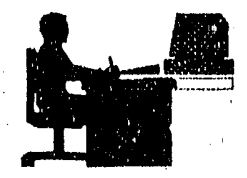

Design
Concurrent Engineering

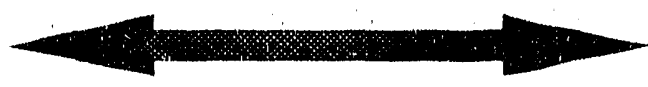

Manufacturing

Analysis'

Test

Application of Workstation Power to:

- Simultaneous Design Collaboration

- Review and Approval Processess

- Intersite Conferencing (Now Dimension: Visualization)

- Collaboration on Other Than Design Documents.

田

SANDIA TECIINOLOCY

QUALITY

Softuare Develippment

Short Development Cycile

Network Integration

T'eamwork

Implementadion de Procedures Development

Cost Reduction

Figure 2 - CLERVER Features

Preliminary tests show that CLERVER generates data streams on the order of $25 \mathrm{~Kb} / \mathrm{s}$. SNL, Division 2813 conducted these particular tests between two DECsystem 3100 workstations connected via $960 \mathrm{~Kb} / \mathrm{s}$ LANmark PBX circuits 131 . We have concluded that the line speed is not a problem with CLERVER. However, beciluse we are concerned about network delays with CLERVER, the NIRVANA network should be free of significant delays to support (TERYER.

\subsection{Traffic Requirements}

We have not quantified NIR VANA traffic requirements. However, we are assuming that NIRVANA will need a high-performance network based on the following facts:

\subsubsection{Number of Workstations}

We expect at least 60 workstations to resull from the first NIR VANA acquisition. However, SNL management has told us to expect up $1050(0)$ workstations within the next three 
NIRVANA Network Requirements

years. We base this estimate on budgetary planning within SNL Organization 20(K). Other organizations have also told us that they expect to participate in the NIRVANA network.

\subsubsection{High-Performance Workstations}

The RFQ also calls for workstations with processing power of at least 8 million instructions/s. We have seen this class of workstation place heavy demands on a network.

\subsubsection{Workstation Configurations}

NIRVANA workstations will be available in two basic configurations. The first configuration will deliver a workstation that is self-contained, including all necessary disk, tape, and memory to operate even withoul a network. We expect this type of workstalion to place little demand on the network.

The second configuration, clusters of diskless workstations, would rely on servers for all disk operations. We have seen diskless workstations place heavy demands on a network.

\subsubsection{File Transfer}

We expect to exchange design files between NIRVANA workstations which are on the order of 8 megabytes in size. Users will exchange these files over the network using either FIP or FTAM. Therefore, we must design the network to accommodate the timely exchange of these files without dramatically reducing the performance of the overall network.

\subsubsection{Role of NFS}

We expect that the Network File System (NFS) will play a significant role in the network. We will likely see most workstations mounting several NIS volumes for applications software and part databases. We expect most workstations to rely heavily on a very few servers for most NFS operations.

\subsection{Access to Other Systems}

NIRVANA users will repuire aceess to certain existing systems at SNL, Albuquerque.

\subsubsection{WhiteStar}

WhiteStar is a parts database for electrical components that most NIRVANA users will need to reference frequently during the design process. White Star currently accommodates terminals using serial protocols over the SNL, data PBX. Eventually, Whitestar will connect terminals using $X$ windows protocols over TCP/IP. WhiteStar is also a secure, unclassified system that is now connected to the 2810 Computer-aided design Integration and Technology network (CITnet). 
NIRVANA Network Requirements

\subsubsection{CADS Network}

The 2800 Computer-Aided Design System (CADS) Network is a production ComputerAided Design (CAD) network that presently serves over 100 CAD workstations for designers in Organization 2850. NIRVANA users must exchange data with users on the CADS Network. Most of this exchange will be between NIRVANA and older, existing electrical CAD systems on the CADS Network. The CADS Network will also give NIRVANA users access to the standard release mechanisms for design information. Currently, the CADS Network is a classified network composed of Digital VAX/VMS systems running DECnet.

\subsection{KCD Requirements}

KCD intends to have 19 (32\%) of the early NIR V NNA workstations with the same basic requirements of any other NIRVANA workstation. However, KCD has specifically requested the CLERVER capability. They have also asked for real-time filc transfer between KCD and SNL using FTAM $|4|$.

\section{Recommended Architecture}

We have proposed a short-term network archilecture in rigures 3, ta, and 4b. This design uses an existing network to satisfy most NIRVANA reguirements in a very short time. The network consists of LANs in each building connected to a fiber-optic backbone at SNL. A secure link also joins the SNL, Albuquerque, NIRVANA network and a NIR VANA LAN at KCD.

\subsection{Role of LANs}

This plan assumes that LANs exist in each building that are joined through a fiber-optic backbone. We know that some local networks exist; however, these LANs must meet Protected Distribution System (PDS) standards before we may connect them to the fiber-optic backbone. We do not know whether all existing LANs meet these standards.

If we do build or change LANs, we suggest that they include SynOptics workgroup concentrators with these alvantages:

A. Multiple Media - The SynOptics 30)() concentrators can accommodate fiber-optic, thin coax, and thick coax media. These concentrators also support unshielded twisted-pair connections using both lattisNet or IEEE standards.

B. Network Management ... The SynOptics network management system is the only known system to provide port-level diagnostics, statistics, and management functions. In addition, SynOprics is delivering a management agent that is compatible with the Simple Network Management Protocol (SNMP). This makes it possible for us to manage all network modules (routers, bridges, concentrators, and workstations) from a single SNMP console. 


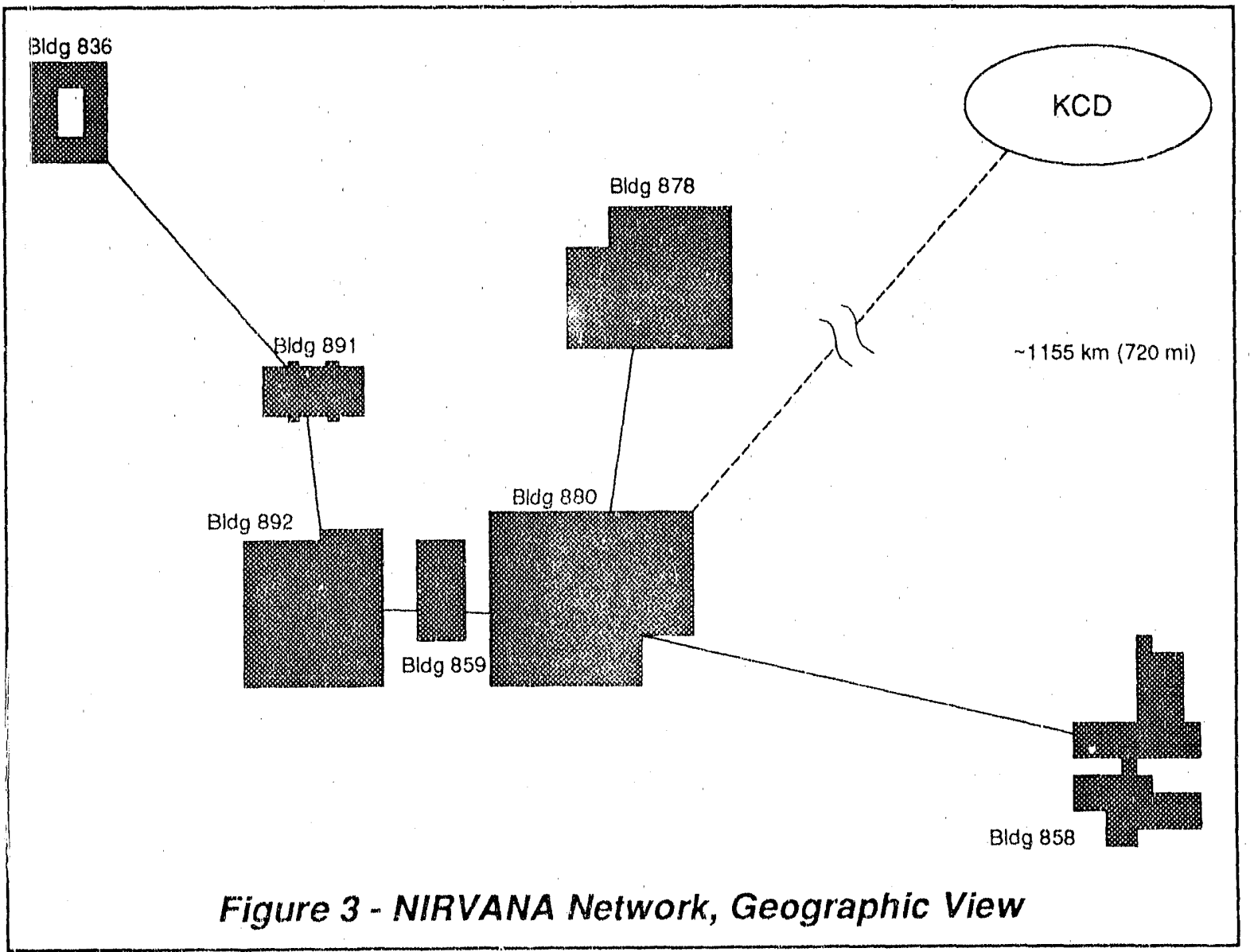

C. Support - Users may contract with at least two local vendors or even SNL Organization 2618 " install and maintain SynOptics equipment at SNL, Albuquerque. In addition, SNL ( rganization 2617 has selected SynOptics as their standard for full bandwidth 1 EEE 802.3 networks at Albuquerque.

D. FDDI - SynOptics has shown their support for the early FDDI standard. FDDI is a key element of the long-term network strategy that we will propose later in Section 6. Therefore, early investments in SynOptics equipment should serve us well for many years.

\subsection{Fiber-Optic Backbone}

Each LAN is linked to the fiber-optic backbone to form a hierarchical network with its root in Building 892. LANs are linked to the backbone with commercial IEEE 802.3 bridges and repeaters. The bridges isolate local traffic to the LANs and also isolate LANs from the distance and loss constraints of the backbone. 


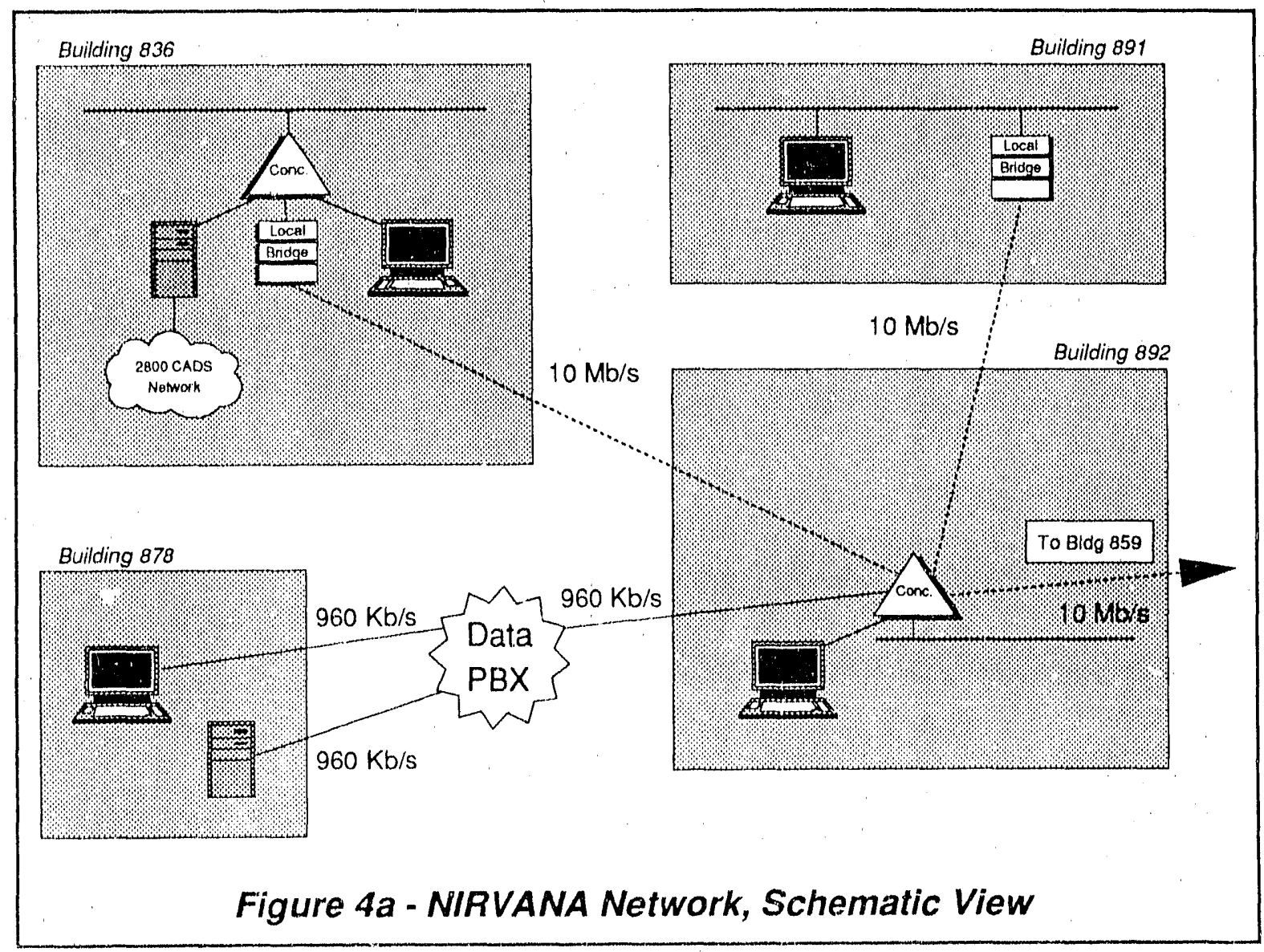

\subsection{SNL Data PBX}

The NIRVANA network also includes a partition of the SNL data PBX which offers the unique LANmark circuits that support IEEE $8(22.3$ links at $960 \mathrm{~Kb} / \mathrm{s}$. SNL Organizations 2617 and 2618 support this capability at no cost to their customers. These links are ideal for connecting single workstations in areas where no LAN exists. However, we do not expect the data $\mathrm{PBX}$ to satisfy our performance requirements for the backbone or for connecting LANs to a fiber-optic backbone.

\subsection{Access to Other SNL Systems}

\subsubsection{WhiteStar}

In this design, we connect WhiteStar to the LAN in Building 859, the most desirable condition. In fact, the new WhiteStar processor, a DECsystem 5810, is currently installed on 


\section{NIRVANA Network Requirements}

the proposed network. Since this system presently uses TCP/IP protocols on an IEEE 802.3 network for its communications, we do not expect to change WhiteStar's communications to satisfy NIRVANA requirements.

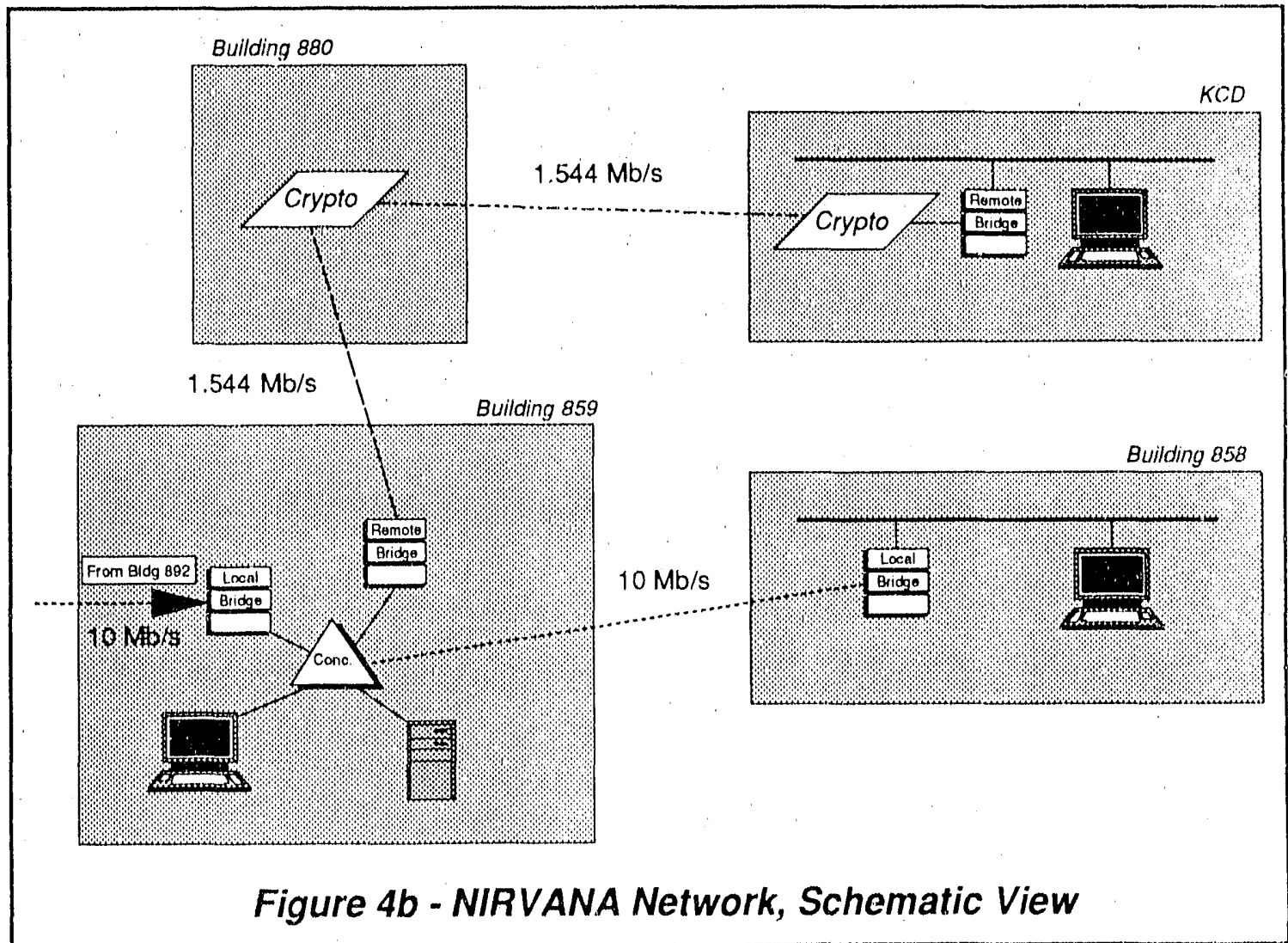

\subsubsection{CADS Network}

We also propose a connection between the 280() CADS Network and NIRVANA in Building 836 where a single VAX processor would link the two networks. This VAX must have two network adapters: (1) for the secure DECnet traffic on the CADS Network, and (2) for unclassified TCP/IP traffic on the NIRVANA network. This system must also run both Digital's security-enhanced Virtual Memory System (SE/VMS) and Wollongong's enhanced WIN/TCP networking software to support this configuration.

SNL Organization 2600 has tested this configuration and tentatively approved it for just such an application. However, DOE must approve any new connection to the CADS Network as a change to the CADS Network Security Plan. 
NIRVANA Network Requirements

\subsection{Link to KCD}

We have also proposed a link between the SNL, Albuquerque, NIRVANA network and a NIRVANA LAN at KCD. Ideally, this link would use a terrestrial T1 line between a pair of remote IEEE 802.3 bridges as shown in Figure $4 \mathrm{~b}$. We should encrypt the part of the link that lies outside secure areas. The link should be carried by terrestrial rather than satellite communications services for the following reasons.

\subsubsection{Minimum Delay}

The full-duplex round-trip delay through a satellite link is about $600 \mathrm{~ms} \mathrm{[5].} \mathrm{There}$ should be no problem tuning TCP/IP traffic for use over a satellite link. However, we will use OSI protocols between SNL and KCD. We do not know if we can tune OSI protocols for these delays.

In addition, KCD has explicitly requested the CLERVER service between KCD and SNL. We are certain that the CLERVER will not work over a satellite link because of the interactive, graphic nature of $\mathrm{X}$ windows applications.

\subsubsection{No OPMODEL at KCD}

The OPMODEL network carries most satellite traffic within the DOE. Because KCD is removing their OPMODEL terminal, using an OPMODEL link to KCD is impractical, or at least very complex and expensive.

\subsection{Benefits}

\subsubsection{Cost}

We could build the proposed network in a short time with little cost. Most elements of the proposed network exist as the Department 2810 CAD Technology and Integration Network. The only new elements of the system would include the links to Buildings 891 , 858,878 , and the link to KCD. Since optical fibers are available in Buildings 891 and 858 , and we expect to use LANmark circuits in Building 878 , the only link that we need to build is the T1 link to KCD.

\subsubsection{Performance}

This design is a high-performance network with most elements being full-bandwidth, IEEE 802.3 equipment. The only delays that we would see on the network are those associated with the T1 link to KCD. A terrestrial T1 link has the least delay of any link that we have considered. If capacity becomes a problem, we can use multiple T1 links between SNL and KCD

\subsubsection{Schedule}

Users can add NIRVANA workstations to the existing network today. Organization 2618 could connect fiber-optic links to Buildings 891 and 858 in as little as six to eight weeks. 
NIRVANA Network Requirements

The only portion of the network that we cannot install quickly is the link to KCD. A T1 line to KCD could take as long as six months to install. Although these estimates may seem reasonable, we must develop a detailed project plan before we have an accurate construction schedule.

\subsection{Caveats}

You must be aware that the proposed network is a near-term solution to the problem of networking early NIRVANA workstations with some limitations.

\subsubsection{No High-Level Management}

This plan simply pronoses a physical network design. We could build such a network easily. However, what happens after we build the network? Who will manage the network?

Network management involves many tasks, for example:

- Node Name and Address Assignments

- User Name and Number Assignments

- NFS Administration

- Mail System Administration

In addition, a network manager must also deal with both TCP/LP and OSI protocols.

Before we build any network, we must develop a network management plan. Otherwise, this network will create more problems than it will solve. We will likely address the network management issues at a later time, in a separate paper.

\subsubsection{Limited Expansion}

It is not a simple matter to add new buildings onto the fiber-optic backbone. Optical fibers must be available to join the new building with another building that is on the existing backbone. These fibers are not available in all buildings.

Although we could add new LANs to the backbone using LANmark circuits, we would prefer to use these circuits only to connect single workstations into the network. We do not expect that the PBX will perform adequately to serve as the backbone or to connect large LANs to a fiber-optic backbone.

\subsubsection{Upgrading CITnet}

We must upgrade CITnet to support NIRVANA workstations. In addition to the need for faster bridges, we must add new SynOptics concentrators to accommodate NIRVANA workstations. These upgrades could cost as much as $\$ 75,000$ in capital funds.

We may also have to upgrade the LANs in Buildings 891, 858, and 878 to meet PDS standards. Upgrading these networks may not be cheap. For example, a suitable SynOptics concentrator can easily cost $\$ 15,000$. Additional costs for installation can drive the cost of a new LAN to as much as $\$ 25,00()$. 


\section{NIRVANA Network Requirements}

We could operate CITnet without some of these upgrades; however, CITrnet cannot meet the performance and function requirements for NIRVANA during the next three years without these improvements.

\subsubsection{Near-Term Solution Only}

Although the proposed network depends heavily on elements of the existing network, we did not design CI'Tnet to satisfy NIRVANA requirements. Indeed, certain elements of CITnet are not suitable for this application, and there are many single points of failure that can cripple the entire network. We cannot remedy these problems without great expense and delay. It is not practical to redesign CITnet to remove these weaknesses. Instead, we will recommend in Section 6 a strategy for developing a new network expressly for NIRVANA.

\subsubsection{Contention}

Several systems and applications presently rely on CITnet. These projects include:

- WhiteStar

- $28(x)$ Secretarial Network

- 2810 Advanced Technology Application Cluster

- 2800) Integrated Office Network Prototype

- SNL Office Automation Working Group Prototype

In addition, Organization 2800 relies on this network as a general purpose, secureunclassified develophent network. We need this capability to accomplish our mission to develop new engineering network technologies.

It is reasonable to expect NIRVANA workstations to share CITnet with these existing applications. It is unreasonable to expect the existing efforts would cease or radically change to accommodate NIRVANA. In fact, many elements of NIRVANA depend on the developmental work that CITnet supports.

\subsubsection{No Classified Processing}

The proposed network will not permit classified processing. Users can exchange only melchsified datu over this network. If anyone uses NIRVANA workstations for classified work, we cannol comnect them to the network.

\subsection{Security Factors}

The goal of this document is to propose a design that is practical and secure. Although we believe that we have succeeded, the SNL. Computing Security establishment may question cerrain elements in this design. 
NIRVANA Network Requirements

\subsubsection{Overall Security Profile}

We have designed a secure-unclassified network that allows only Q-cleared personnel to have access to the network. In addition, we will limit network access to secure areas at SNL and KCD. We also cannot allow auto-answer telephone modems or the exchange of any classified or private data on this network.

\subsubsection{Other Traffic}

This network must support TCP/IP and OSI protocols for NIRVANA workstations. Since CITnet nodes currently use DECnet, LAT, and XNS protocols, we must expect the NIRVANA network to handle all of these different network protocols.

\section{4:8.3 KCD Link}

We have proposed a link to KCD (Figure 4b) which will connect a NIRVANA LAN at KCD with the SNL NIRVANA network. We expect to encrypt the link outside secure areas. Only KCD NIRVANA workstations will have access to the NIRVANA network through this link.

\subsubsection{CAD Network Access}

We have proposed that a VAX connect the classified 280) CADS Network to the unclassified NIRVANA network. The VAX would use SE/VMS and secure Wollongong WIN/TCP to enforce mandatory access controls. SNL Organization 260) has tested this configuration for use on the SNL. Secure Supercomputing Network (SSN). We plan to apply these same principals to a CADS/NIRVANA link.

\section{Alternatives}

As we prepared this paper, we examined two alternatives to the proposed network.

\subsection{PBX-Based System}

We could use the SNL, data PBX as the NIRVANA backbone as shown in Figure 5. Here, we connect LANs and single workstations to a single partition of the PBX via LANmark cir. cuits. The main advantage to building this type of network is that it is readily available at no cost.

The only problem with the PBX is the performance of the LANmark circuits. At $960 \mathrm{~kb} / \mathrm{s}$, the LANmark circuits are not suitable for all connections. For example, if all 6() NIRVANA workstations mount a common NFS volume from a single server (Figure 5), all must conlend for a single $960 \mathrm{~kb} / \mathrm{s}$ line to use this server. 'This would be an unacceptable condition that causes problems even on $10 \mathrm{Mb} / \mathrm{s}$ networks. Nevertheless, this is exactly how we plan to use the network. 


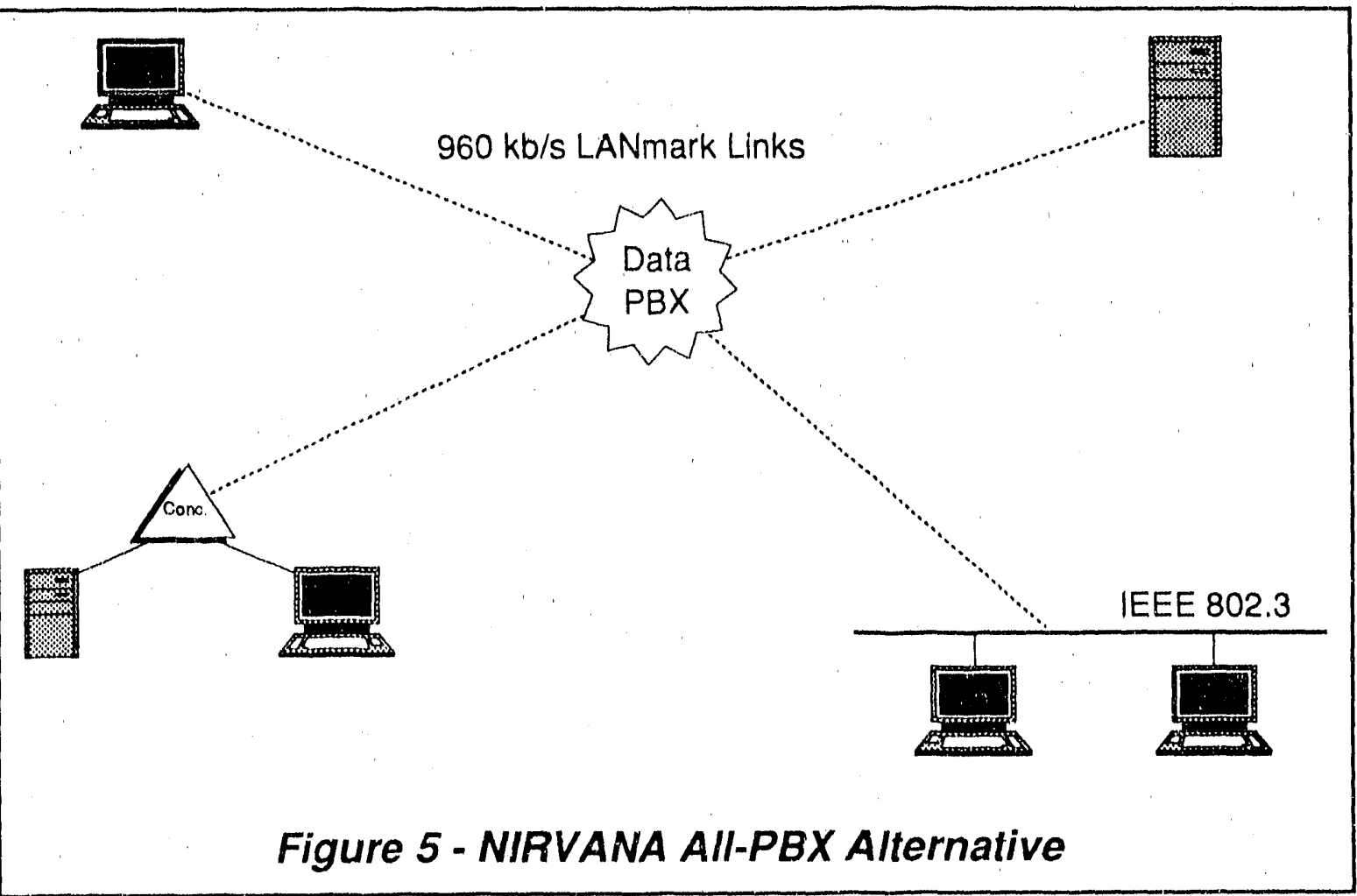

\subsection{SSN Connection}

NIRVANA could also join the SNL Secure Supercomputing Network (SSN). Here, NIRVANA workstation would connect to TCP/IP routers on the SSN through LANmr $\mathrm{k}$ circuits (Figure 6). The main advantage to this network is that it is the only approved metnod to network classified and unclassified TCP/IP systems.

There are several disadvantages to becoming SSN subscribers.

- Costs - SNL Organization 2600 will charge a fee for all rodes connecting to the SSN. This fee, about $\$ 5,(0) 0$ a node/yr, for 60 workstations could amount to $\$ 300,000 / \mathrm{yr}$.

- No OSI - Network connections to the SSN are made to TCP/IP routers. Since OSI traffic does not traverse the routers, OSI protocols could not be used on the SSN.

- Network Management - The SSN is heavily managed. Organization 2600's provision for managing the SSN could actually turn out to be an advantage for the NIRVANA consortium. Thus, the management service alone may be worth $\$ 5,000$ per workstation. 


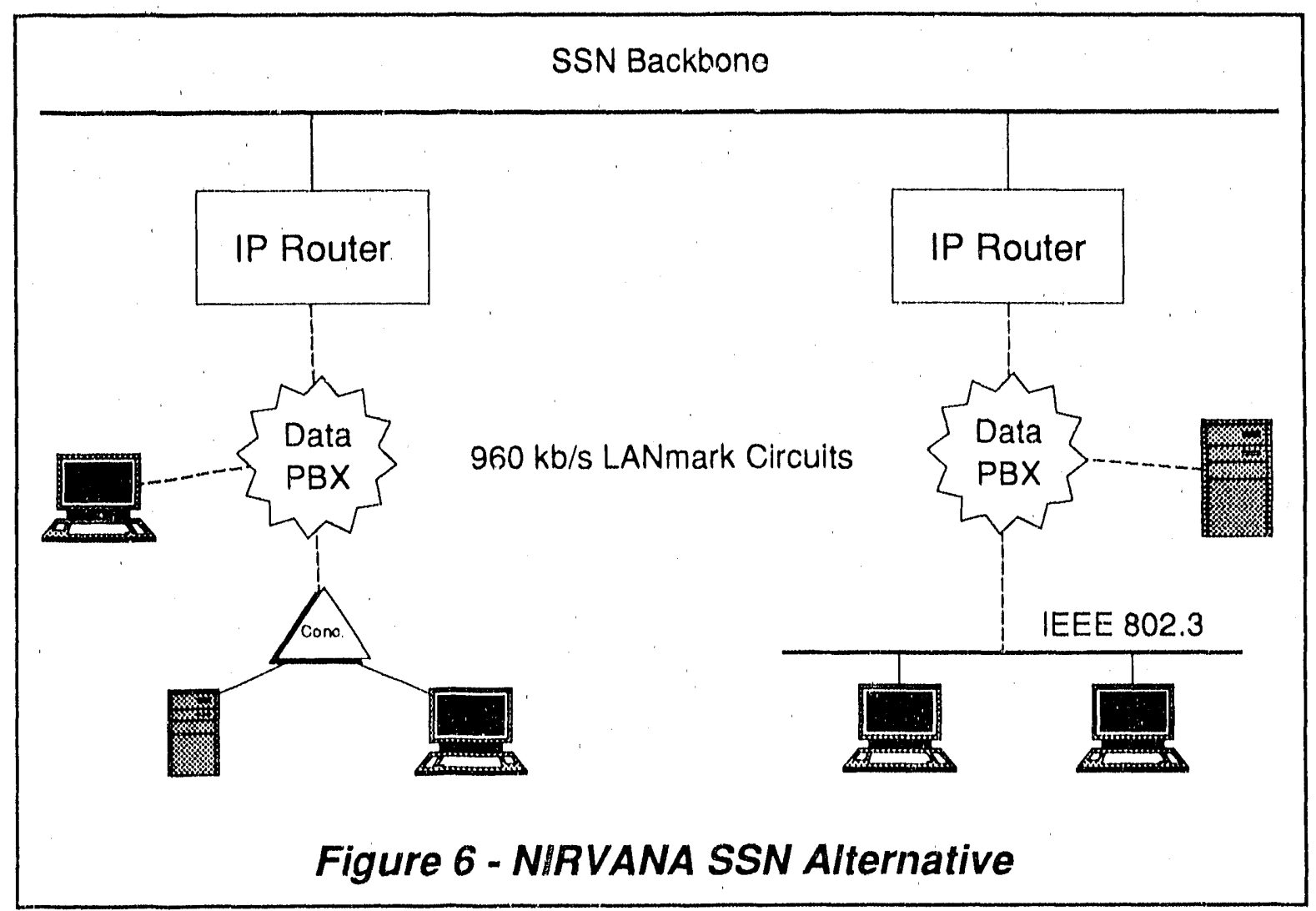

- Kerberos - The SSN uses Kerberos to enforce authentication on the network. Workstations must have the Kerberos added to their normal network software, and Organization 2600 has added Kerberos to many workstations. However, if NIRVANA selects a workstation that Organization 2600 has not already modified, it may be difficult and time-consuming to add the Kcrberos code to NIRVANA 7 workstations.

- Lack of Services - Some network services, such as electronic mail, will not be supported throughout the SSN.

- LANmark Connection - All connections to the SSN routers are made through LANmark circuits on the SNL data PBX. As a result, a network that uses the SSN as its backbone inherits all the problems of the SNL data PBX.

The SSN is not a desirable base for a NIRVANA network. However, the SSN is the only viable solution if NIRVANA has both classified and unclassified workstations. 
NIRVANA Network Requirements

\section{Long-Term Strategy}

We huve stated that the proposed network would satisfy our short-term needs. However, we expect NIRVANA to become larger and more critical to the mission of the Nuclear Weapons Complex. For this reason, we feel that we must begin now to develop a network that can satisfy all NIR VANA requirements in the future.

We have proposed a long-term NIRVANA network architecture in Figure 7. Here, LANs could . ise both FDDI and IEEE $8(2.3$ technologies. These high-speed $(100 \mathrm{Mb} / \mathrm{s})$ LANs are then connected to a centrel switching node via optical fibers. The links between the LANs and the switch could be FDDI, DS-3 (45 Mb/s), or some other high-speed interface.

FIDDI equipment is presently available from some network vendors, although most industry analysts do not expect FDDI to become practical for another three years [6]. In addition, SNL

intends to buy a high-speed switchino, sys em for this type of application. We do not expect this switch to be available at SNL for at least two more years.

Therefore, we propose that the NIRVANA consortium begin now to develop this highperformance network. Given planning and budgeting cycles, today is the right time to begin this effort.

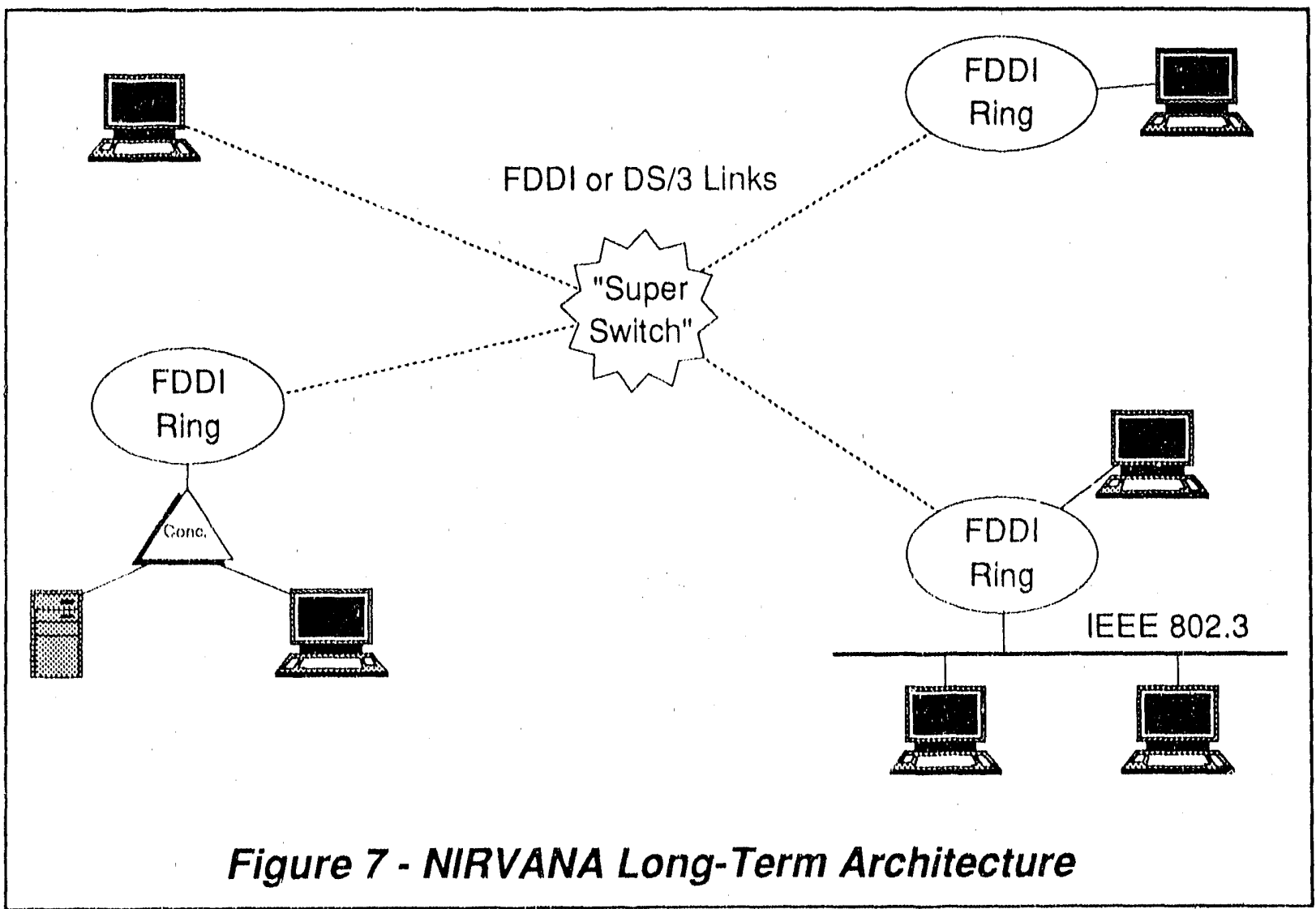


NIRVANA Network Requirements

\section{Conclusions}

We have examined the requirements for networking NIRVANA workstations. From these requirements, we propose a near-term solution using an existing network in Department 2810 . Division 2813 could allapt this network to support NIRVANA workstations in a short time with minimal costs.

The proposed near-term network would be a secure-linclassified network with unclassified links to both the KCD and the $28(0)$ CADS Network. Department 2810 will continue to use this network to support their network development efforts.

We examined two alternatives. First, we considered using the SNL data PBX for a network backbone. This solution is inexpensive, but the LANmark circuits do not deliver the needed performance. Second, we considered using the SSN as a backbone for NIRVANA, which would give us many problems. However, it is the only system that networks both classified and unclassified workstations.

We have also proposed a long.term network for NIRVANA. The design would use FDDI technologies to provide a secure, high-performance network that meets all NIRVANA requirements. This network will take at least three years to build. 
NIRVANA Network Requirements

\section{References}

1. US Department of Commerce/National Bureau of Standards, Federal Information Standard publication (FIPS PUB) 146: Government Open Systems Interconnection Profile (GOSIP), National Technical Information Service, US Department of Commerce, Springfield, VA, 1988.

2. Dean, C., An X Window-Based Collaboration Facility, Sandia National Laboratories, Albuquerque, NM, To be Published.

3. Tolendino, L. F., Nelson, S. D., Gossage, S. A., and Bickel, D. L., Operating Experience with the Sandia Terminal Switching Network and Loop, Sandia National Laboratories, Albuquerque, NM (SAND 88-3472C), 1988, Available from NIST.

4. Nirvana Video Conference - The KCD network requirements were established during a video conference. Those attending from SNL included Jim Yoder, Brad Wood, and John Naegle. Those attending from KCD included Ken Schmidt, Scott Pohl, and. Craig Parrish. Albuquerque, NM and Kansas City, MO, 5/14/90).

5. Feher, K., Digital Communications, Satellite/Earth Station Engineering, Prentice-Hall Inc., Englewood Cliffs, NJ, 1981, p 16.

6. Heywood, P., and Greenfield, D., "FDDI: Just Say Not Yet," Data Communications Magazine, McGraw-Hill, New York, NY, April 1990, pp 4.5-48. 

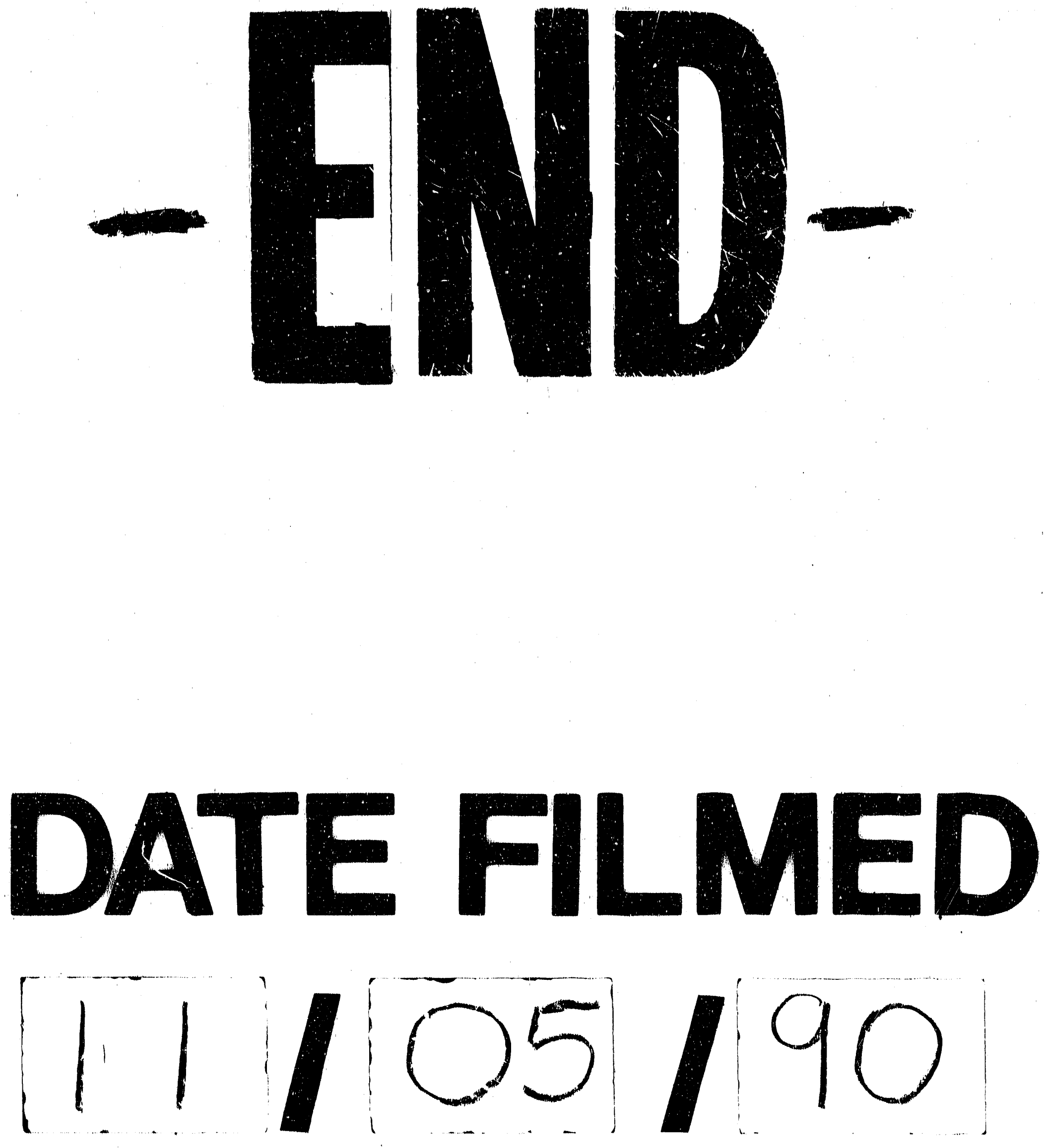
